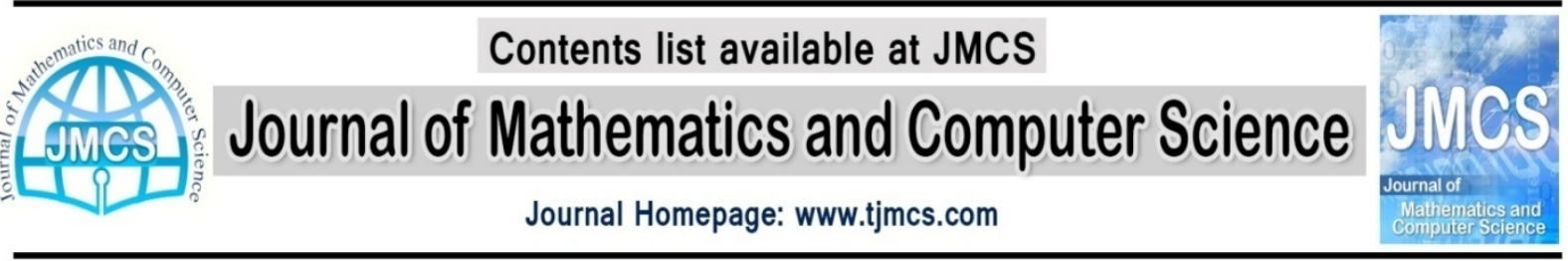

\section{Effects of Nimodipinee on cerebral hemodynamic and prognosis of diffuse axonal injury patients with repeated measurements design}

\author{
Kianoush Fathi Vajargah*1, Robabe Mehdizadeh $^{2}$ \\ ${ }^{1,2}$ Department of Statistics, Islamic Azad University, North branch, Tehran, Iran \\ k_fathi@iau-tnb.ac.ir, mehdizadeh18@yahoo.com
}

Article history:

Received February 2013

Accepted March 20013

Available online April 2013

\begin{abstract}
In medical and behavioral researches experimental units, are often the people who are in different social status, physical and other tendency So their responses will be different. In these situations they are required to control the changes in the result of this sources that are potentially variable otherwise the source of variability can increase mean square error significantly and make impossible revealing the real difference between treatments. Appropriate model for such research projects are repeated measurements.

This study is a prospective study that accesses the effects of Nimodipinee on Hemodynamic changes of cerebral vessels and the short time prognosis in DAI patients. The study was done in Trauma word of Imam Reza hospital of Tabriz Iran.

Forty DAI patients were randomized in two equals groups the case group underwent treatment with Nimodipine drug (every 4 hours after admission) Control groups did not received this treatment.

Hemodynamic changes in these patients were measured using transcranial Doppler machine on the first third and tenth days of admission.
\end{abstract}

Using a repeated measurements design fitting model 


$$
Y_{i j k}=\mu+\tau_{i}+\beta_{j(i)}+\gamma_{k}+(\tau \gamma)_{i k}+(\beta \gamma)_{k j(i)}+\varepsilon_{m(i j k)}
$$

In which $\tau_{\mathrm{i}}$ is the drug effect (groups Case and Control) is fixed and is in two levels. $\mathrm{B}_{\mathrm{j}(\mathrm{i})}$ is the effect Of $\mathrm{j}$-th patient in group $\mathrm{i}$, which is in twenty level. $\gamma_{\mathrm{k}}$ is TCD stages that is fixed and it is in three levels. $(\tau \gamma)_{\mathrm{ik}}$ and $(\beta \gamma)_{\mathrm{kj}(\mathrm{i})}$ are correspond interactions, this finding obtained that there is no significant differences in Doppler variables between three time TCD and Nimodipine did not make significant difference between the variables.

In this study using Nimodipine in DAI patients did not make significant effect on Doppler variables. Therefore, using this drug for this group of patients is not recommended.

Keywords: repeated measurements design, Nimodipine, patients with DAI

\section{Introduction}

In medical and behavioral research of often experimental units, the of people due to certain social status, physical and other areas they may there answer is very different. In this situations are required to contra the changes in the result of this source that are changeable the potential for this changeable to controlled. Because in the otherwise the source of variability can increase mean square error significantly to reveal be impossible in the real difference between treatments. Appropriate plan for such research projects are repeated measurements. In this study, the effects of Nimodipinee on cerebral hemodynamic and prognosis of diffuse axonal injury patients has been analyzed using this method.

Diffuse axonal injury (DAI), Vazvaspasm and cerebral ischemia are progressive events which therapeutic interventions can improve the prognosis of them in the courses $(1,2,3)$. Diffuse axonal injury (DAI) is a common pattern of brain injury, which is often underestimated. Traffic accidents are the main cause of DAI. DAI is the most important traumatic brain lesion leading to impairment of consciousness and late disability $(1,3,4)$.

For traumatic and aneurismal $\mathrm{SAH}$, Nimodipinee has become a standard treatment (1) Nimodipine acts on spastic vessels selectively and improves blood flow 
in ischemic areas (5). Vazvaspam and brain ischemia are other important events that are seen in traumatic patients.

Tran cranial Doppler (TCD) is a non-invasive method, permitting measurement of blood flow velocity in cerebral arteries (6). A few studies assessing the effectiveness of nimodipine on DAI patients. Thus, the present investigation was designed to study hemodynamic changes in DAI patients and the effects of nimodipine on Doppler variable and the patient's outcome.

\section{Materials and methods}

This study is a prospective clinical trial and a double blind study that accesses the effects of Nimodipine on hemodynamic changes of cerebral vessels and the short time prognosis in DAI patients. The clinical study period was in the trauma ward of Imam Reza hospital of Tabriz, Iran. Patients of both sexes, aged above 12, with brain CT findings of DAI were enrolled. In this study, standard exclusion criteria were followed.

Forty patients were included in this trial, which were randomized in two equal groups. The case group underwent treatment with Nimodipine $60 \mathrm{mg}$ every 4 hours via gavages in the first 12 hours of their admission. The control group didn't receive this treatment. Both groups underwent bedside TCD studies in the days 1, 3 and 10 of their admission. Peak systolic velocity (PSV), end diastolic velocity (EDV), mean flow velocity (MFV), and plasticity index (PI) of both middle cerebral arteries (MCA) were recorded. Normal MFV in MCA is $62 \pm 12 \mathrm{~cm} / \mathrm{sec}(1,6)$.

\section{Results}

The study population consisted of 40 patients (32 males and 8 females) with age range of 12 to 70 years (28.25 \pm 14.08). The case group included 20 patients (16 male, 4 female) with the mean age of $26.90 \pm 12.88$. There were no significant differences between the case and control groups about age and sex. The most common cause of DAI in our cases was traffic accidents (80\%). 
With fitting model

$$
Y_{i j k}=\mu+\tau_{i}+\beta_{j(i)}+\gamma_{k}+(\tau \gamma)_{i k}+(\beta \gamma)_{k j(i)}+\varepsilon_{m(i j k)}
$$

For mean flow velocity (MFV) in which $\tau_{\mathrm{i}}$ treatment effect (groups Case and Control) is fixed and has two levels. $\mathrm{B}_{\mathrm{j}(\mathrm{i})}$ is the effect of $\mathrm{j}$-th patient in group $\mathrm{i}$ which is in 20 levels and randomized. $\gamma_{\mathrm{k}}$ is TCD stages that is fixed and has three levels. $(\tau \gamma)_{\mathrm{ik}}$ and $(\beta \gamma)_{\mathrm{kj}(\mathrm{i})}$ are correspond and interactions.

Here because of the lack of repetition in each stages of TCD, error is not calculable. Range of indices in general, $\mathrm{i}=1,2, \ldots$, a and $\mathrm{j}(\mathrm{i})=1,2, \ldots, \mathrm{b}$ and $\mathrm{k}=1,2$, ..., $\mathrm{c}$ and $\mathrm{m}=1,2, \ldots, \mathrm{n}$ allocate and here: $\mathrm{a}=2, \mathrm{~b}=20, \mathrm{c}=3$ and $\mathrm{n}=1$.

Analysis of variance with separation of total sum squares:

$$
\begin{gathered}
\sum_{i} \sum_{j} \sum_{k}\left(\bar{y}_{i j k}-\bar{y}\right)^{2}=\sum_{i} \sum_{j} \sum_{k}\left[\left(\bar{y}_{i j}-\bar{y}\right)+\left(y_{i j k}-\bar{y}_{i j}\right)\right]^{2} \\
=c \sum_{i} \sum_{j}\left(\bar{y}_{i j}-\bar{y}\right)^{2}+\sum_{i} \sum_{j} \sum_{k}\left(y_{i j k}-\bar{y}_{i j}\right)^{2} \\
(\text { abc-1) }=(a b-1)+\quad(\text { abc-ab })
\end{gathered}
$$

Total Between patients within the patients Difference between patients

$$
\mathrm{Df}=\mathrm{ab}-1=39
$$

Patient internal difference Df $=a b(c-1)=40(2)=80$ and both of them are independent.

Sum squares between patients, including both changes related to difference of groups and differences of patient within Groups. The two can be divided as follows.

$$
\begin{aligned}
c \sum_{i} \sum_{j}\left(\bar{y}_{i j}-\bar{y}\right)^{2} & =c \sum_{i} \sum_{j}\left[\left(\bar{y}_{i}-\bar{y}\right)+\left(\bar{y}_{i j}-\bar{y}_{i}\right)\right]^{2} \\
=b c \sum_{i}\left(\bar{y}_{i}-\bar{y}\right)^{2} & +c \sum_{i} \sum_{j}\left(\bar{y}_{i j}-\bar{y}_{i}\right)^{2} \\
(\mathrm{ab}-1) & =(\mathrm{a}-1)+\mathrm{a}(\mathrm{b}-1)
\end{aligned}
$$

Between patients treatment within Patients 
Sum squares of treatment with a- $1=1$ digress of freedom and sum squares of patients within treatment a (b-1) = 38 digress of freedom are independent from together. Sum squares or internal changes of patients including changes related to TCD stage, interaction effect TCD stage* treatment and interaction effect TCD stage * the patient within treatment.

We separate these components as follows:

$$
\begin{aligned}
& \sum_{i} \sum_{j} \sum_{k}\left(y_{i j k}-\bar{y}_{i j}\right)^{2}=\sum_{i} \sum_{j} \sum_{k}\left[\left(\bar{y}_{k}-\bar{y}\right)+\left(\bar{y}_{i k}-\bar{y}_{i}-\bar{y}_{k}+\bar{y}\right)\right. \\
& \left.+\left(y_{i j k}-\bar{y}_{i . k}-\bar{y}_{i j}+\bar{y}_{i}\right)\right]^{2}=a b \sum_{k}\left(\bar{y}_{k}-\bar{y}\right)^{2}+b \sum_{i} \sum_{k}\left(\bar{y}_{i k}-\bar{y}_{i}-\bar{y}_{k}+\bar{y}\right)^{2} \\
& +\sum_{i} \sum_{j} \sum_{k}\left(y_{i j k}-\bar{y}_{i k}-\bar{y}_{i j}+\bar{y}_{i}\right)^{2}
\end{aligned}
$$

This sum squares respectively with $\mathrm{c}-1=2$, (c-1) (a-1) $=2$ and a (b-1) (c-1) $=76$ digress of freedom are independent from each other.

According to the statistical model can be obtained such partition for the total sum squares, too. If in the statistical model instead of each effect we put its estimation we obtain:

$$
\begin{aligned}
& y_{i j k}=\mu+\left(\mu_{i}-\mu\right)+\left(\mu_{i j}-\mu_{i}\right)+\left(\mu_{i k}-\mu_{i}-\mu_{k}+\mu\right) \\
& +\left(\mu_{i j k}-\mu_{i j}-\mu_{i k}+\mu_{i}\right)+\left(y_{i j k}-\mu_{i j k}\right)
\end{aligned}
$$

and the result of putting samples means rather than populations means is:

$$
\begin{aligned}
& y_{i j k}=\bar{y}+\left(\bar{y}_{i}-\mu\right)+\left(\bar{y}_{i j}-\bar{y}_{i}\right)+\left(\bar{y}_{i k}-\bar{y}_{i}-\bar{y}_{k}+\bar{y}\right) \\
& +\left(y_{i j k}-\bar{y}_{i j}-\bar{y}_{i k}+\bar{y}_{i}\right)
\end{aligned}
$$


If we move $\mathrm{y}_{\ldots .}^{-}$to the left side and square two sides can get after the summing in values of $\mathrm{i}, \mathrm{j}$ and $\mathrm{k}$ and considering that multiply two by two are zero:

$$
\begin{aligned}
& \sum_{i} \sum_{j} \sum_{k}\left(\bar{y}_{i j k}-\bar{y}\right)^{2}=b c \sum_{i}\left(\bar{y}_{i}-\bar{y}\right)^{2}+c \sum_{i} \sum_{j}\left(\bar{y}_{i j}-\bar{y}_{i}\right)^{2}+a b \sum_{k}\left(\bar{y}_{k}-\bar{y}\right)^{2} \\
& +b \sum_{i} \sum_{k}\left(\bar{y}_{i k}-\bar{y}_{i}-\bar{y}_{k}+\bar{y}\right)^{2}+\sum_{i} \sum_{j} \sum_{k}\left(y_{i j k}-\bar{y}_{i j}-\bar{y}_{i k}+\bar{y}\right)^{2}
\end{aligned}
$$

Sum of values that are required to calculate the sum of squares are determined in table 1.

\begin{tabular}{|c|c|c|c|c|c|}
\hline group & patiant & Firest TCD & Second TCD & Third TCD & sum \\
\hline & 1 & 74 & 69 & 64 & 207 \\
\hline & 2 & 79 & 79 & 63 & 221 \\
\hline & 3 & 116 & 67 & 45 & 228 \\
\hline & 4 & 55 & 56 & 50 & 161 \\
\hline & 5 & 66 & 58 & 61 & 185 \\
\hline & 6 & 53 & 51 & 48 & 152 \\
\hline & 7 & 40 & 71 & 74 & 185 \\
\hline & 8 & 64 & 65 & 50 & 179 \\
\hline \multirow[t]{21}{*}{ Case } & 9 & 50 & 58 & 63 & 171 \\
\hline & 10 & 61 & 66 & 66 & 193 \\
\hline & 11 & 43 & 64 & 63 & 170 \\
\hline & 12 & 95 & 89 & 88 & 272 \\
\hline & 13 & 82 & 95 & 75 & 252 \\
\hline & 14 & 67 & 71 & 45 & 183 \\
\hline & 15 & 48 & 47 & 40 & 135 \\
\hline & 16 & 45 & 79 & 42 & 166 \\
\hline & 17 & 45 & 59 & 55 & 159 \\
\hline & 18 & 45 & 103 & 55 & 203 \\
\hline & 19 & 66 & 64 & 43 & 173 \\
\hline & 20 & 74 & 72 & 80 & 226 \\
\hline & & 1268 & 1383 & $\mathbf{1 1 7 0}$ & 3821 \\
\hline & 21 & 55 & 77 & 42 & 174 \\
\hline & 22 & 55 & 75 & 53 & 183 \\
\hline & 23 & 103 & 71 & 80 & 254 \\
\hline & 24 & 50 & 43 & 61 & 154 \\
\hline & 25 & 96 & 69 & 53 & 218 \\
\hline & 26 & 111 & 124 & 115 & 350 \\
\hline & 27 & 109 & 128 & 117 & 354 \\
\hline & 28 & 83 & 67 & 39 & 189 \\
\hline
\end{tabular}

Table 1. 


\begin{tabular}{cccccc}
\hline Control & 29 & 56 & 50 & 50 & 156 \\
& 30 & 140 & 93 & 132 & 365 \\
& 31 & 69 & 64 & 48 & 181 \\
& 32 & 24 & 82 & 55 & 161 \\
& 33 & 64 & 63 & 87 & 214 \\
34 & 96 & 66 & 74 & 236 \\
& 35 & 56 & 101 & 71 & 228 \\
& 36 & 45 & 57 & 53 & 155 \\
37 & 50 & 50 & 50 & 150 \\
& 38 & 64 & 71 & 74 & 209 \\
39 & 164 & 96 & 95 & 355 \\
& 40 & 75 & 72 & 53 & 200 \\
\hline & sum & $\mathbf{1 5 6 5}$ & $\mathbf{1 5 1 9}$ & $\mathbf{1 4 0 2}$ & $\mathbf{4 4 8 6}$ \\
\hline & & & & \\
\hline & Total & 7985 & 7430 & 23617 & 39032 \\
& sum & & & \\
\hline
\end{tabular}

Between patients

$S S=c \sum_{i} \sum_{j}\left(\bar{y}_{i j}-\bar{y}\right)^{2}=\sum_{i} \sum_{j} \frac{y^{2}{ }_{i j}}{c}-\frac{y^{2}}{a b c}=\frac{207^{2}+221^{2}+\ldots+200^{2}}{3}-\frac{8307^{2}}{120}$

$=45534.59$

Treatment

$S S=b c \sum_{i}\left(\bar{y}_{i}-\bar{y}\right)^{2}=\sum_{i} \frac{y_{i}{ }^{2}}{b c}-\frac{y^{2}}{a b c}=\frac{3821^{2}+4486^{2}}{60}-\frac{8307^{2}}{120}=3658.21$ treatment

$$
\begin{aligned}
& S S=c \sum_{i} \sum_{j}\left(\bar{y}_{i j}-\bar{y}_{i}\right)=\sum_{i}\left(\sum_{j} \frac{y_{i j}{ }^{2}}{c}-\frac{y_{i}^{2}}{b c}\right) \\
& =\left(\frac{207^{2}+\ldots+226^{2}}{3}-\frac{3821^{2}}{60}\right)+\left(\frac{174^{2}+\ldots+200^{2}}{3}-\frac{4486^{2}}{60}\right)=41876.38
\end{aligned}
$$

within

Patients

Within

patients

$$
\begin{aligned}
& S S=\sum_{i} \sum_{j} \sum_{k}\left(y_{i j k}-\bar{y}_{i j}\right)^{2}=\sum_{i} \sum_{j} \sum_{k} y_{i j k}{ }^{2}-\sum_{i} \sum_{j} \frac{y_{i j}{ }^{2}}{c} \\
& =74^{2}+\ldots+53^{2}-\frac{207^{2}+\ldots+200^{2}}{3}=20551.34
\end{aligned}
$$


Stage TCD

$$
\begin{aligned}
& S S=a b \sum_{k}\left(\bar{y}_{k}-\bar{y}\right)^{2}=\sum_{k} \frac{y_{k}^{2}}{a b}-\frac{y^{2}}{a b c} \\
& \left(\frac{2833^{2}+2902^{2}+2572^{2}}{40}-\frac{8307^{2}}{120}\right)=1514.85
\end{aligned}
$$

treatment*TCD stage

$$
\begin{aligned}
& S S=b \sum_{i} \sum_{k}\left(\bar{y}_{i k}-\bar{y}_{i}-\bar{y}_{k}+\bar{y}\right)^{2}=\sum_{i} \sum_{k} \frac{y_{i k}{ }^{2}}{b}-\frac{y^{2}}{a b c} \\
& -{ }_{\text {treatment }} S S-{ }_{\text {stageofTCD }} S S=\frac{1268^{2}+\ldots+1402^{2}}{20}-\frac{8307^{2}}{120}-3658.21-1514.85 \\
& =328.02
\end{aligned}
$$

TCD stage* treatment within patients (group)

$$
\begin{aligned}
& S S=\sum_{i} \sum_{j} \sum_{k}\left(y_{i j k}-\bar{y}_{i j}-\bar{y}_{i k}+\bar{y}_{i}\right)^{2} \\
& =\sum_{i} \sum_{j} \sum_{k} y_{i j k}{ }^{2}-\sum_{i} \sum_{j} \frac{y_{i j}{ }^{2}}{c}-\sum_{i} \sum_{k} \frac{y_{i k}{ }^{2}}{b}+\sum_{i} \frac{y_{i}{ }^{2}}{b c}=18708.47
\end{aligned}
$$

Table 2. ANOVA table to the right middle cerebral artery MFV (MCA)

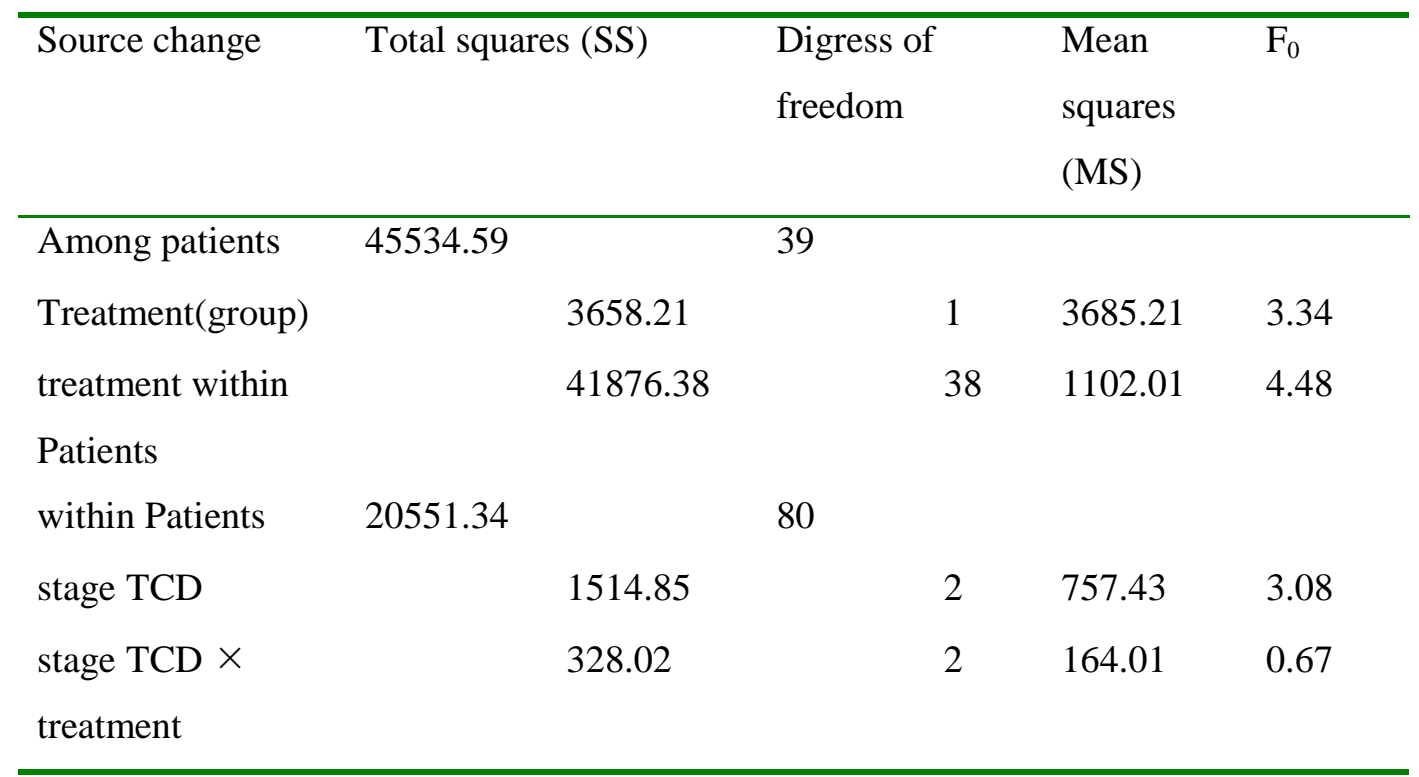




\begin{tabular}{llll}
\hline stage TCD $\times$ & 18708.47 & 76 & 246.16 \\
treatment within & & & \\
Patients & & & \\
\hline total & $66112 / 93$ & 119 & \\
\hline
\end{tabular}

Compared with obtained values for the F0 with their analogous amount in distribution points table $\mathrm{F}_{0.05, \mathrm{v} 1 \text {, v2 }}(10)$, when $\mathrm{F}_{0}<\mathrm{F}_{0.05 \text {, v1, v2 }}$ be noted that none of the sources of change are not significant.

In this study, repeated measurements ANOVA models has been done as above and also using Minitab statistical software for factors pulsatility index (PI), mean flow velocity (MFV), peak systolic velocity (PSV) and end diastolic velocity (EDV) in both internal carotid artery and the right and left sides of middle cerebral a arteries (MCA). Nimodipine did not cause any significant effect on those variables.

\section{Discussion}

DAI was termed by Adams in 1982 and considered as the main cause of morbidity and mortality of head injury (4).

Nimodipine decreases neurological morbidity, improves the outcome and it is a safe drug $(7,8)$. The hemodynamic effects of nimodipine treatment can be monitored by the use of serial TCD investigations (6). In the literature, we found only one study about the effects of nimodipine on DAI patients. According to this case-control studylusion, which assessed 89 patients with DAI, nimodipine could not create a significant difference between the two groups (1).

Repeated measurements design that is used in this study for analyzing the data is known as a within subjects design in which all of the subjects attend in every experimental conditions of the project that can be one of the weaknesses of these designs. Because in some research projects the inclusion of one subjects in all conditions of the research is impossible (9).

Repeated measurements design makes researchers be able to measure how the subjects are changing with passing the time. 


\section{Conclusion}

In this study using Nimodipine in DAI patients did not make significant effect on Doppler variables. Therefore according to our results by analyzing the data, we don't have strict recommendation $\mathrm{f}$ or using this drug for this group of patients. Although this effect was not significant, but the view that Nimodipine is a safe drug had beneficial effects of the patients from clinical view in improvement and was well tolerated by patients and reduced vasospasm not prohibited in these patients.

\section{References}

[1] Feng D, Ma Y. zhaag Y. Ching Tromatol. 3(2): pp-85-889(2000).

[2] Youmans JR. , Neurological surgery, $4^{\text {th }}$ ed. Saunders, Philadelphia, 543-546, 15621564,1651-1662(1996).

[3] Wesserman JR, Koenigsberg RA . Diffuse axonal injury.(http:/www.emedicine.com/radio topic 216.htm) [Accessed 9 May 2004].

[4] Jiang Shu, Ju Y, He Y, He M, Mao B. Chin J Traumatol. 4(4): pp-204-7(2001).

[5] Yang Sy, Wang Zg. Chin J Traumatol, 6(6): pp-326-31.(2003)

[6] Van Santbrink H, Schouten JW, Ssteyberg EW Avezaat CJ, Maas AI. Acta Neurochir (Wein), 144(11): pp-1141-1149(2002).

[7] Czosnyka M, Smiclewski P, Kirkpatrick P, Menon DK, Pickard JD. Stroke, 27:pp-18291834(1996).

[8] Yang JQ, Zhou QX. Acta Pharmacol Sin. 22(5): pp-423-427(2001).

[9] http://en.wikipedia.org/wiki/Repeated measures design

[10] Montgomery DC, Peak EA. Introduction to linear regression analysis, $2^{\text {nd. }}$ ed. Shahid Bahonar University of Kerman. (1992).

[11] M. Bagheri, M. Valipour, V. Amin, The Bankruptcy Prediction in Tehran share holding using Neural Network and it's Comparison with Logistic Regression,5(3),pp-219228.(2012).

[12] A. Arjmandzadeh, S. Effati, Interval Support Vector Machine in Regression Analysis, 2(3), pp- 565- 571 (2011).

[13] A.H. Hadjahmadi, T. J. Askari, A Decision Support System for Parkinson's Disease Diagnosis using Classification and Regression Tree, 4(2) pp-257 - 263,(2012). 